\title{
In-hospital outcome of patients with peripartum cardiomyopathy - a single center study
}

\section{Silvana Jovanova1*, Frosina Arnaudova- -Dezulovic', Marjan Boshev', Vesna Livrinova ${ }^{2}$, Lidija Palasheva², Iva Miceva ${ }^{3}$}

'University Clinic of Cardiology, Skopje, Macedonia

2University Clinic of Gynecology and Obstetrics, Skopje, Macedonia

${ }^{3}$ University Clinic of Cardiology, Medical Faculty, Skopje, Macedonia
RECEIVED:

September 21, 2014
KEYWORDS: peripartum cardiomyopathy, systolic dysfunction, heart failure, prognosis.

CITATION: Cardiol Croat. 2014;9(9-10):437.

*ADDRESS FOR CORRESPONDENCE: University Clinic of Cardiology, Vodnjanska 17, 1000 Skopje, Macedonia. Phone: +389-72231116 / E-mail: silvana_jovanova@yahoo.com

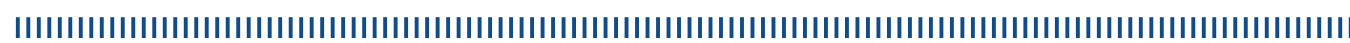

Peripartum cardiomyopathy (PPCMP) is a disorder of unknown cause in which initial left ventricular systolic dysfunction and symptoms of heart failure (HF) occur between the last month of pregnancy and the first 5 months postpartum. The causes and pathogenesis are poorly understood and PPCMP remains a diagnosis of exclusion. Clinical presentation includes usual signs and symptoms of heart failure, and unusual presentations relating to thromboembolism. Effective $\mathrm{HF}$ treatment reduces mortality rates and increases the number of women who fully recover left ventricular (LV) systolic function. PPCMP is common in some countries and rare in others.

During 6 years period (2008-2014) 22 patents (pts) were admitted to our hospital with diagnosis of PPCMP. 16(73\%) pts were admitted to our hospital immediately post-partum, because of acute HF which needed immediate and aggressive HF treatment. Six (27\%) pts presented congestive HF symptoms during the first three months after delivery. At the admission all the pts had documented LV systolic dysfunction with echocardiography $(E F<45 \%)$. Five of them $(23 \%)$ had severely impaired LV systolic function (EF=25-30\%), seven (32\%) pts had EF of 30-40\%, and $10(45 \%)$ pts had mild LV systolic dysfunction. During the hospitalization, clinical and functional improvement and stabilization was achieved in all patients Complete recovery of LV systolic function was observed in 12 (55\%) pts with PPCMP. In 10 pts (45\%) there was persistence of LV systolic dysfunction determined with echocardiography.

Peripartum cardiomyopathy is rare, but a serious disease associated with significant cardiac functional deterioration Early diagnosis and appropriate medical treatment allows good functional recovery in majority of these patients and favorable prognosis. Reliable population-based information about incidence and prevalence of PPCMP is essential to the development of health policies for prevention and control of this condition.

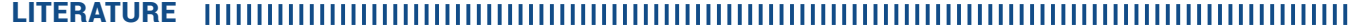

1. Fett JD, Christie LG, Carraway RD, Murphy JG. Five-year prospective study of the incidence and prognosis of peripartum cardiomyopathy at a single institution. Mayo Clin Proc. 2005;80(12):1602-6.

2. Johnson-Coyle L, Jensen L, Sobey A. Peripartum cardiomyopathy: review and practice guidelines. Am J Crit Care. 2012;1:89-98. 